\title{
Simulation of Magnetic Field Penetration of Cylindrical Cavity with Wound Solenoid
}

\author{
Baoquan Mao, Tu Lan ${ }^{*}$ and Wei Deng \\ Department of Weapon Engineering, Academy of Armored Force Engineering, Beijing 100072, China \\ ${ }^{*}$ Corresponding author
}

\begin{abstract}
In order to generate a magnetic field with adjustable magnetic field intensity and high uniformity, a magnetic field simulation model of the cylindrical cavity of wound solenoid was established by Ansoft Maxwell 3D, by changing the external magnetic field excitation source frequency, the steady-state conditions and low-frequency conditions of the magnetic field of the cylindrical cavity magnetic field distribution characteristics. The results show that the excitation frequency of the magnetic field source is less than $100 \mathrm{~Hz}$, the intensity of the magnetic field after penetration is $61.9 \%$ of the excitation magnetic field intensity, and the intensity of the magnetic field decreases exponentially with the increase of the excitation frequency.
\end{abstract}

Keywords-alternating electromagnetic field; Maxwell 3D; simulation model; solenoid

\section{INTRODUCTION}

The application of magnetic field cylindrical cavity in a wide range of applications. In the military field, the electromagnetic field generated by the barrel of the field gun can not only transmit energy, improve the performance of the projectile ${ }^{[1-2]}$, but also transmit information and improve the detection of the barrel ${ }^{[3]}$, In recent years, magnetic field has been studied deeply in the field of gun barrel defect detection, fuze induction energy storage and electromagnetic coil gun firing. However, artillery barrels are usually made of ferromagnetic material, high permeability and eddy current effect will cause the alternating magnetic field is difficult to penetrate the wall into the chamber, literature [2] pointed out that the titanium tube outside the excitation frequency of less than $5 \mathrm{Khz}$ magnetic field, the barrel wall to retain the external excitation field strength of $80 \%$. So the magnetic field penetration problems to be solved.

In electromagnetism, for the infinite length, current-carrying round wire and close-wound finite-length solenoid has been related to the theoretical derivation and numerical calculation[4],However, most researches on the magnetic field of the solenoid are focused on the constant magnetic field, while the research on the alternating magnetic field is less, and most of them are calculated and analyzed theoretically. While the study of electromagnetic field penetration process only solved a few cases, Such as low-frequency magnetic field penetration detection of thin steel sheet thickness[5]; cylindrical and rectangular solid separation of variable method solution[6-7].

So far, no study has been conducted on the electromagnetic transmission characteristics of the cylindrical cavity in the solenoid. the model of the electromagnetic field of the cylinder cavity is established, and the electromagnetic induction characteristics of the cavity are discussed. The cylindrical cavity is taken as an example and the finite element simulation model is established based on Ansoft Maxwell software.

\section{Finite ElEMENT SimUlation Model OF THE ESTABLISHMENT AND ANALYSIS}

\section{A. Electromagnetic Field Analysis Method}

Electromagnetic field numerical analysis methods are differential and integral method[8];Differential method also includes finite difference and finite element analysis methods, while the difference method is not suitable for the boundary conditions are complex, irregular boundary conditions; The finite element method is a numerical method based on variational principle partition interpolation. It divides the solution domain into many small units, solves an approximate solution for each unit, and then derives the global solution of the solution domain. The more precise meshing, the less distortion area, the more accurate the solution.

Ansys Maxwell software is used to analyze the electromagnetic field of the spiral wound cylindrical cavity. The main steps are as follows[9]:

(1) Pre-treatment: the establishment of models, given the material properties, meshing, boundary conditions and load (excitation);

(2) Solve: Set the number of convergence steps, time step, percentage of fault tolerance;

(3) Post-processing: View the magnetic field lines, magnetic field strength calculation results.

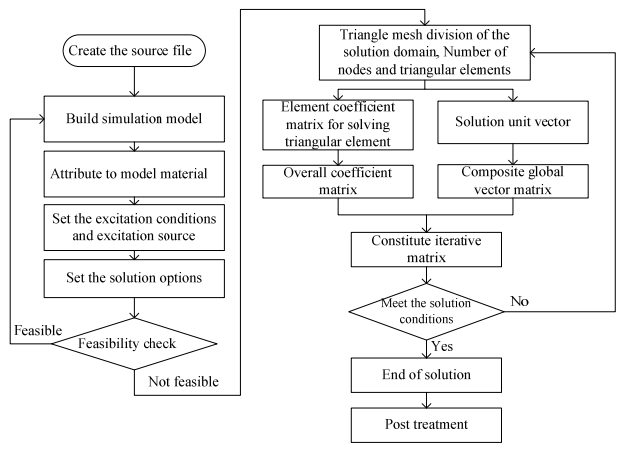

FIGURE I. MAXWELL 3D SIMULATION PROCESS 


\section{B. The Establishment Of Simulation Model}

The simulation model shown in Figure 2, the external ring for the excitation coil, the internal cylinder for the barrel model, the barrel length is much larger than the magnetic field, it can be regarded as infinite barrel length. In the model, the tube length is $600 \mathrm{~mm}$, the outer diameter is $40 \mathrm{~mm}$, the inner diameter is $30 \mathrm{~mm}$, and the inner diameter of the solenoid is $35 \mathrm{~mm}$.Solenoid coil diameter $50 \mathrm{~mm}$, diameter $40 \mathrm{~mm}$, Length of $300 \mathrm{~mm}$; dense around the outer wall of the cylinder, located in the middle of the empty length, cylinder material used PCrNi3MoVA steel, the solenoid material is set to copper.

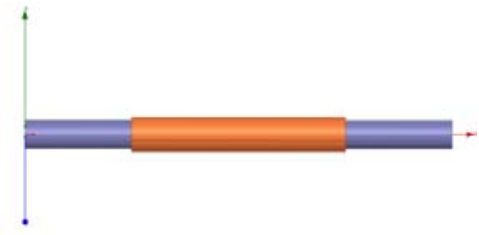

FIGURE II. SIMULATION MODEL OF CYLINDRICAL CAVITY WITH CLOSE-WOUND SOLENOIDS

For the alternating magnetic field, and the magnetic field has a high permeability of the metal components, magnetic field module should be used in the eddy current solver, select the Eddy current Magnetic, coordinates Cartesian coordinate system, the length of the unit $\mathrm{mm}$. The tangential component of the magnetic field intensity and the normal component of the magnetic flux density are continuous under natural boundary conditions, so the interface between the objects is the natural boundary condition. Neumann boundary conditions, the magnetic field strength is tangent to the boundary surface, and the magnetic field intensity is zero in the normal component, this paper does not study the radial magnetic field distribution, so all the boundary conditions defined as Neumann boundary conditions[10].Set the solution area to air, arbitrarily divide a cross section across the solenoid model, and apply a current excitation to add the solution settings. Figure 3 is the result of the manual division, the maximum length of the tube grid is defined as $35 \mathrm{~mm}$, the maximum length of the coil grid is $20 \mathrm{~mm}$.

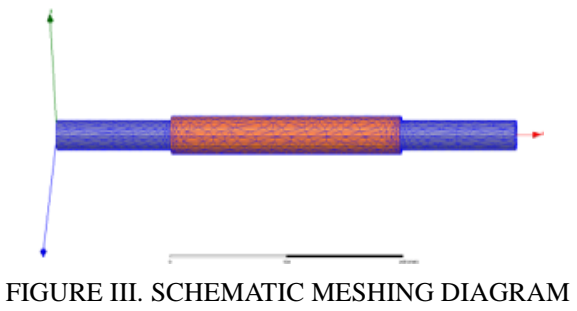

\section{Results Analysis}

After setting the excitation and boundary conditions, the model check, and then calculate the solution, the final model of the magnetic field strength vector, magnetic field strength and magnetic field diagram.

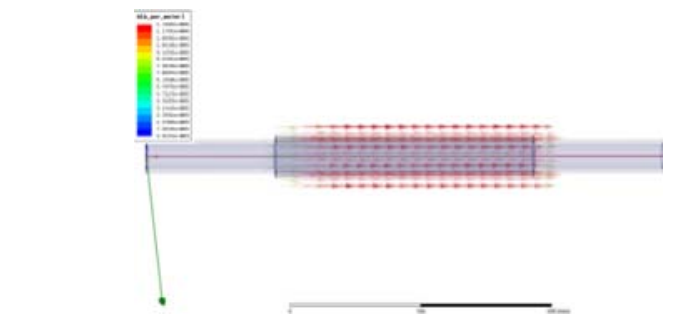

FIGURE IV. MAGNETIC FLUX DENSITY VECTOR DIAGRAM OF CYLINDRICAL CAVITY

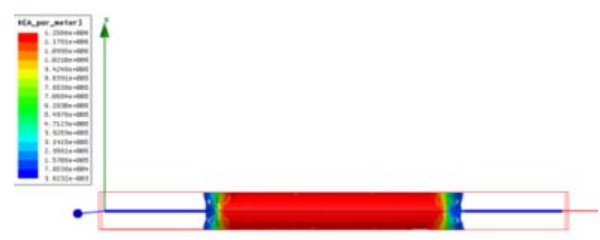

FIGURE V. THE MAGNETIC FLUX DENSITY OF THE SOLENOID

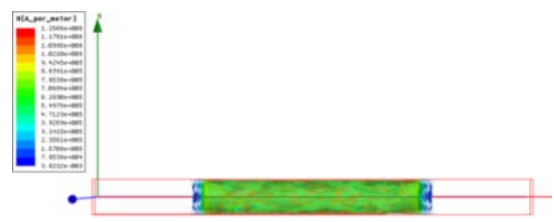

FIGURE VI. THE MAGNETIC FLUX DENSITY OF THE CYLINDER WALL

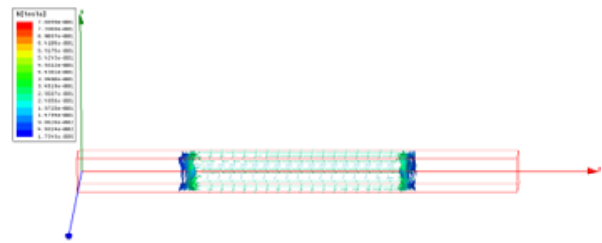

FIGURE VII. CLOUD DENSITY OF THE MAGNETIC FLUX DENSITY INSIDE THE CYLINDER CHAMBER

From Figure 4 to Figure 7 , it can be seen that the magnetic field is mainly concentrated in the solenoid portion and the magnetic induction intensity outside the solenoid coil length range is low. The strength of the magnetic field across the solenoid is significantly higher than that of the cylindrical wall, and the magnetic field strength of the wall is significantly higher than that of the interior of the chamber, indicating that the presence of the metal cylinder changes the spatial magnetic field of the solenoid Configuration, reducing the cylindrical cavity inside the magnetic field strength. 


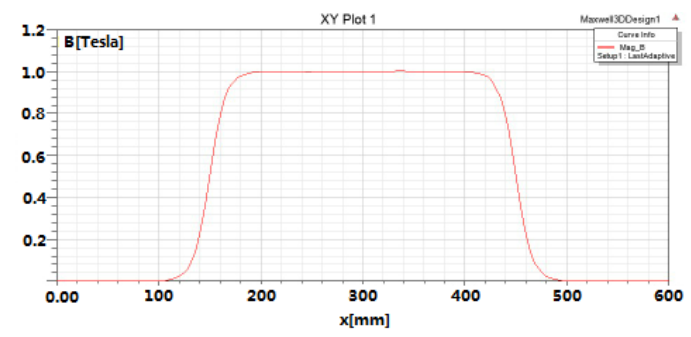

FIGURE VIII. DISTRIBUTION OF THE MAGNETIC FIELD ALONG THE AXIS WHEN THERE IS NO CONDUCTOR IN THE SOLENOID

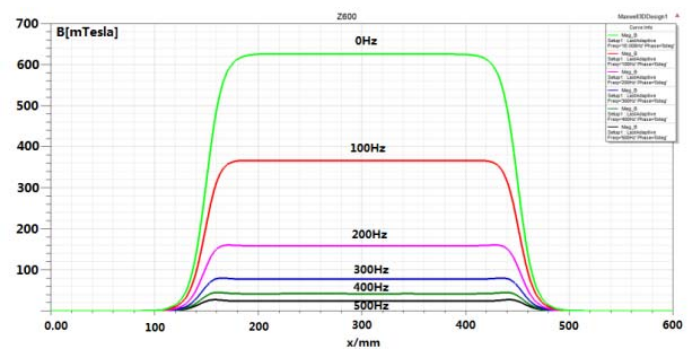

FIGURE IX. VARIATION OF MAGNETIC FIELD AT DIFFERENT FREQUENCIES

Figure 8 for the cylindrical cavity at the axis of the magnetic induction intensity curve, As can be seen from the figure, the cylindrical cavity in the solenoid part of the magnetic field along the axis of uniform distribution, the end of the rapid attenuation of the solenoid. Figure 9 shows the magnetic flux density of the cylindrical cavity at the magnetic field frequencies of $0 \mathrm{~Hz}, 100 \mathrm{~Hz}, 200 \mathrm{~Hz}, 300 \mathrm{~Hz}$ and $500 \mathrm{~Hz}$, respectively. Figure 8 and Figure 9, compared with the steady-state magnetic field curve, we can see in the metal conductor in the solenoid, the magnetic field is significantly reduced. Metal conductor under the excitation of alternating magnetic field, resulting in eddy current, eddy current electric field generated reverse magnetic field, so the metal conductor weakened the magnetic field in its internal space distribution. When the excitation frequency is $100 \mathrm{~Hz}$, the intensity of the magnetic field after penetration is $61.9 \%$ of the excitation magnetic field, while the excitation frequency reaches $500 \mathrm{~Hz}$, the magnetic field after the penetration is only $3.4 \%$ of the excitation magnetic field intensity.

\section{CONCLUSION}

(1) alternating magnetic field in the metal cylinder in the eddy current, and the vortex will produce the magnetic field through the metal cylinder weakened. When the magnetic field frequency is small, the eddy current in the metal is also small, easy to penetrate the magnetic field, the larger the magnetic field in the chamber, on the contrary, the higher the frequency, eddy current stronger magnetic field penetration more difficult The simulation results show that the external excitation frequency is less than $100 \mathrm{~Hz}$, the intensity of the magnetic field after penetration is $61.9 \%$ of the excitation magnetic field intensity, and the intensity of the magnetic field is exponentially decaying with the increase of the excitation frequency.

(2) the use of close-wound solenoid of the magnetic way to obtain a more uniform axial magnetic field distribution.
However, because each coil produces a strong magnetic field requires a high excitation current, so the actual production of strong magnetic field is difficult, in addition to the magnetic permeability of steel material is much larger than the air, so most of the magnetic lines through the metal tube wall, The internal magnetic field of the cylindrical wall is much larger than the internal magnetic field of the cavity. Therefore, the spatial distribution of coil magnetic field in addition to the coil itself parameters, the magnetic flux distribution in space to its greater impact. To study the spatial magnetic field configuration of the solenoid, we need to consider these two factors.

\section{REFERENCES}

[1] Li Jun, Yan Ping, Yuan Weiqun. Electromagnetic Gun Technology and Its Development[J]. High Voltage Engineering, 2014,04: 1052-1064.

[2] Wu Zhiliang, Zhang He.The Magnetic Field Penetration Characteristics in bore of Small Caliber Fuse Using Inductive Energy[J].Acta Armamentarii, 2010,10: 1310-1315.

[3] Guo Xiling. Research on technology of Alternative current Magnetic Field Leakage and Flaw Identification to the Whorl[D]. National University of Defense Technology, 2005.

[4] Feng Tao. Analysis on Field Distribution Characteristics of Limited Solenoid[D]. Xi'an University of Electronic Science and Technology, 2013.

[5] Huang Ping. Application Research of Low Frequency Electromagnetic Technology in Defect Detection of Tank Bottom[D]. Shenyang University of Technology, 2016.

[6] FU Kang, LIN Chun-sheng, ZHOU Jian-jun.The simulation of internal magnetic field of the infinite cylinder shielding cavity[J] .Ship Science and Technology, 2013,08: 96-98.

[7] DENG Hai, HU Xian-Quan, JIANG Ming-Yu, HUANG Jun.A Study of the Eddy Current Distribution inside a Conductor Cylindrical Boundary Surface for Quasi-steady-state[J].Journal of Chongqing Normal University (Natural Science Edition), 2004,03: 28-30.

[8] Wang Deqiang, Yu Qiang.Overview on the numerical calculation method of ship's magnetic field[J] .Ship Science and Technology, 2014,03: 1-6.

[9] Xiong Lan, Wu Yimei, Yang Zikang, Shi Lingling, Miao Xuefei, He Wei.Electromagnetic Field Simulation Analysis and Experiment on Processing Chambers of High-Frequency Electromagnetic Water Treatment Devices[J].High Voltage Engineering, 2012, 02: 427-434.

[10] Tang Ying. Research on Defect Detection based on Pulsed Magnetic Flux Leakage Field Nondestructive Testing Technology[D]. National University of Defense Technology, 2011. 